\title{
Common Knowledge Promotes Cooperation in the Threshold Public Goods Game by Reducing Uncertainty
}

\author{
Paul Deutchman ${ }^{1 *}$, Dorsa Amir ${ }^{1}$, Matthew Jordan², Katherine McAuliffe ${ }^{1}$ \\ 1. Boston College, Department of Psychology, Chestnut Hill, MA 02467, United States of \\ America \\ 2. Yale University, School of Management, New Haven, CT 06511, United States of \\ America
}

\begin{abstract}
Recent work suggests that an important cognitive mechanism promoting coordination is common knowledge-a heuristic for representing recursive mental states. Yet, we know little about how common knowledge promotes coordination. We propose that common knowledge increases coordination by reducing uncertainty about others' cooperative behavior. We examine how common knowledge increases cooperation in the context of a threshold public goods game, a public good game in which a minimum level of contribution-a threshold-is required. Across three preregistered studies $(\mathrm{N}=5,580)$, we explored how varying (1) the information participants had regarding what their group members knew about the threshold and (2) the threshold level affected contributions. We found that participants were more likely to contribute to the public good when there was common knowledge of the threshold than private knowledge. Participants' predictions about the number of group members contributing to the public good and their certainty ratings of those predictions mediated the effect of information condition on contributions. Our results suggest that common knowledge of the threshold increases public good contributions by reducing uncertainty around other people's cooperative behavior. These findings point to the influential role of common knowledge in helping to solve large-scale cooperation problems.
\end{abstract}

Keywords: Cooperation, Public Goods Game, Thresholds, Common Knowledge, Certainty 


\section{Introduction}

Cooperation is a key aspect of human social life. While adaptations supporting cooperation are found in many organisms (Clutton-Brock, 2009), cooperation among humans stands out in both its scale and scope. Humans are unique in the extent to which we cooperate with unrelated individuals (Axelrod \& Hamilton, 1981; Fehr \& Fischbacher, 2004; Rand \& Nowak, 2012) and we cooperate in group sizes that are unmatched in the animal kingdom (Clutton-Brock, 2009). While these features of human cooperation have undoubtedly contributed to our success as a species, they also point to a key question: what are the psychological mechanisms that enable humans to solve cooperation problems of this scale?

\subsection{Common knowledge}

One cognitive mechanism that plays an important role in cooperative behavior is common knowledge (sometimes called mutual knowledge; Baltag et al., 2016; Clark \& Marshall, 1981; Halpern \& Moses, 1990; Rubinstein, 1981). Common knowledge is the recursive belief state in which $A$ knows $X, B$ knows $X, A$ knows that $B$ knows X, B knows that A knows that B knows X, ad infinitum. Recent work suggests that common knowledge is an important mechanism for coordinating group behavior (Thomas et al., 2014; Thomas et al., 2018). For example, past work has found that people were more willing to attempt risky coordination when there was common knowledge about the mutually beneficial joint payoff for coordination compared to when there was only shared knowledge (such as secondary and tertiary knowledge states; Thomas et al., 2014). These results suggest that common knowledge is likely a distinct cognitive state that may have evolved to solve recurrent problems in human social life (DeFreitas et al., 2019). While previous work suggests that common knowledge plays a role in coordinating behavior (Thomas et al., 2014), no work has explored exactly how it does so. What are the mechanisms that underlie the effect of common knowledge on cooperation?

Much of the work on economic games that model cooperation assumes that actors have complete information about the task, that is, they have common knowledge. However, in many circumstances, including more ecologically valid contexts, decision makers lack access to complete information regarding the boundaries and payoffs of the cooperative interaction. Uncertainty about the structure of the task, and importantly, uncertainty about others' knowledge about the task, tends to negatively affect cooperative behavior (Marks \& Cronson, 1999; McBride, 2010; Wit \& Wilke, 1998). Therefore, a possible mechanism that might explain why common knowledge increases cooperation is that it decreases uncertainty about the social interaction, and specifically, uncertainty about other agents' cooperative behavior. In other words, common knowledge may increase cooperation because it increases certainty that other group members will also contribute when doing so is mutually beneficial, thereby reducing the chance that a cooperative actor will be exploited by others who act selfishly.

\subsection{Threshold Public Goods Game}


An economic game that is well-suited for studying whether common knowledge increases cooperation is the threshold public goods game. The threshold public goods game is a variant of the public goods game (PGG). In the standard PGG, participants are given an endowment and placed into groups that can vary in size across different instantiations of the game (Fehr \& Gachter, 2000; Fischbacher et al., 2001). Participants can contribute any portion of their endowment to a common pot. All contributions to the common pot are then multiplied by the experimenter by a value greater than one and divided equally amongst all group members regardless of their contributions. This game thus captures an important and recurrent dilemma when it comes to cooperation: the conflict between what is best for the individualfreeriding by not contributing anything while others contribute-and what is best for all the members of the group-everyone contributing the entirety of their endowment, resulting in the largest group payoff.

In the threshold PGG, groups must reach a certain level of collective contributions - a threshold-in order for the common pot to be multiplied by the experimenter (Fischbacher et al., 2001). The threshold PGG captures an important feature of many real-life coordination problems - namely, that in many cases, a certain level of contributions must be reached before there are benefits to the initial investment. Take, for example, the case of barbasco fishing, a subsistence practice found among many indigenous Amazonian groups (Heizer, 1953). Barbasco fishing involves the diffusion of a piscicide made from local plants into a river or stream to poison and catch fish. This practice involves multiple contributors who play discrete yet complementary roles, such as building a dam, preparing the barbasco poison, spreading it into the river, herding the fish, and spearing or scooping them. If too few people join in to fulfill the necessary roles, the enterprise will likely be unsuccessful, in which case the initial investment of time and energy will have been wasted. However, it seems probable that if a critical mass of contributors is reached, such that there are enough contributors to fulfill all necessary roles, the chances of success are likely to dramatically increase. This example demonstrates that for certain recurrent social problems, and especially those relevant to our fitness in the evolutionary past, the threshold PGG can be considered a more ecologically valid game than the standard PGG.

Introducing a threshold alters the structure of the game, changing it from a social dilemma-where the interest of the individual is in conflict with the interests of the group - to more of a coordination problem (such as the stag hunt or assurance game; Jansson \& Eriksson, 2015; Skyrms, 2003)—where there are multiple stable equilibria (Archetti \& Scheuring, 2012). In the case where all members have to contribute to meet the threshold, it is in every actor's best interest to contribute to the public good, but only if the other players contribute as well. However, when the threshold is at an intermediate level, for example when half of the group must contribute to meet the threshold, the game becomes an anticoordination problem. In an anti-coordination problem, the stable equilibrium is a mixed strategy, such that the best strategy for an actor is to anti-coordinate with other members by withholding their contribution if the other group members contribute, and investing in the public good if other groups members withhold their contributions (Hauert \& Doebeli, 2004). Thus, when the threshold is at an 
intermediate level, the game resembles the volunteer's dilemma, in which there is a strong incentive to free ride but if everyone defects, all players lose (Diekmann, 1985). ${ }^{1}$

The threshold PGG is an ideal economic game to study common knowledge because contributing in the task more closely models coordination than the standard PGG. Since we know that common knowledge increases coordination, it is expected that common knowledge of a threshold will also increase contributions in this task. This is supported by the foundational work of Schelling on coordination problems which suggests that people often rely on focal points-salient features in a coordination problem - to help solve coordination problems (Schelling, 1960). When there is common knowledge, thresholds might constitute a type of focal point that facilitates coordination by reducing uncertainty about others' behavior. In relation to the example of barbasco fishing above, common knowledge that a certain number of contributors is necessary to catch fish might reduce uncertainty about whether other people will contribute, allowing individuals to coordinate on the mutually beneficial outcome.

To date, no work has explicitly investigated common knowledge in the threshold PGG, nor the mechanism through which common knowledge increases coordination. We predict that common knowledge will increase cooperation in this task by reducing uncertainty about whether your group members will also contribute, and thus whether players will succeed in reaching the threshold. Putting ourselves in the mind of a player, the logic is as follows: when I know that we all know the threshold (and that everyone knows that everyone knows), I can be more confident that everyone will contribute, which will in turn make me more likely to contribute myself. Alternatively, it is possible that our beliefs about others' cooperative behavior, and our certainty in those beliefs, will not influence our own cooperative behavior. In other words, people may behave cooperatively or selfishly without regard to other agents' knowledge about the threshold or beliefs about how they will behave. This question has important implications for real-world cooperation problems as it is often the case that we are uncertain about what others know and will do in cooperative endeavors. That we do not yet know whether certainty mediates the effect of common knowledge on coordination represents an important gap in our understanding of social cognition and cooperation.

Furthermore, while previous work has examined common knowledge in the context of a 2-player stag hunt game (Thomas et al., 2014)-a coordination problem in which the stable strategy is mutual cooperation or defection-no work has examined common knowledge in n-player cooperation problems which more closely model the kinds of cooperation problems we encounter in everyday life.

\subsection{Present study}

\footnotetext{
${ }^{1}$ Importantly, contributing in the threshold PGG, as it more closely models coordination or anticoordination problems, is distinct from pure cooperation-in which an actor contributes a benefit at a cost to themselves (West et al., 2007). However, for ease of comprehension and continuity, we describe contributions in the threshold PGG as cooperation in the sense that they confer a benefit to the group.
} 
In three studies, we explored how common knowledge and threshold levels influenced contributions in a threshold PGG and whether the effect of common knowledge of the threshold on contributions is mediated by certainty about others' cooperative behavior. In Study 1, we tested this by manipulating 1) the information group members knew regarding the threshold, and 2) the level of threshold needed to receive the public good. Because past work has found mixed results regarding the effect of threshold size on contributions (Andrews et al., 2019; Cadsby \& Maynes, 1999), we varied the threshold level in our studies to examine if threshold size predicts contributions and to explore whether the effect of common knowledge varies by threshold size or is robust across different sized thresholds. If thresholds promote cooperation, as found previously, we would expect contributions to be higher in all threshold conditions than in the baseline PGG that lacks a threshold. If common knowledge allows individuals to coordinate contributions in the PGG, then we would expect the highest levels of contributions in the common knowledge condition. In our second preregistered study (Study 2), we aimed to replicate our findings from Study 1 and to test whether the effect of common knowledge on contributions in the threshold PGG is mediated by certainty about the predicted number of group members contributing to the public good. If common knowledge of the threshold increases contributions by reducing uncertainty around the cooperative behavior of others, then we expect to find that contributions in the PGG are mediated both by the predicted number of group members contributing, and certainty about those predictions. Lastly, in a third preregistered study, we aimed to replicate the results of Study 2 and to better understand contribution behavior under common ignorance, in which participants know there is a threshold but do not know what it is, to determine whether certainty about the presence of a threshold might explain the contributions levels.

\section{Study 1}

\subsection{Participants}

We tested $\mathrm{N}=2,252$ participants (52.35\% female), aged 18-77 $(\mathrm{M}=36.93)$ from Amazon's Mechanical Turk in a preregistered study (http://aspredicted.org/blind.php?x=63ct9y). Our sample size was based on previous work on threshold public good games (Jordan et al., 2017) using the same platform. While we initially recruited 3,365 participants, 475 were excluded from analysis for failing to complete the study in its entirety, 618 were excluded for failing the comprehension questions, and an additional 20 were excluded for responding with "three or more" to a question assessing the number of questions they answered without reading or thinking about them carefully. The high rates of exclusions here reflect our stringent exclusion criteria for the several comprehension checks participants had to answer (see preregistration for exclusion criteria). 


\subsection{Design}

Study 1 was a $3 \times 3$ between-subjects design in which participants were randomly assigned to one of three information conditions (common knowledge, common ignorance, and private knowledge) and one of three threshold levels (low, high, and maximum) or a baseline condition with a standard PGG with no threshold. Thus, in total, participants were assigned to one of ten conditions. We used ex-post matching to randomly assign participants to groups of four and determine their group contributions after data collection (Horton, Rand, \& Zeckhauser, 2011; Jordan, McAuliffe, \& Rand, 2016).

\subsection{Procedure}

Participants provided informed consent and were given instructions for the task that detailed the rules of the PGG. Participants were assigned to groups of six and allocated an endowment of $\$ 0.30$ each for the PGG. They were informed of their group size, that all contributions to the public pot would be multiplied by two and divided evenly amongst group members, and they would receive this endowment as a bonus after completing the game.

After reading the instructions, participants answered three comprehension questions to ensure their understanding of the game (see Supplement). Participants who failed the comprehension checks after two attempts were excluded from analyses. After answering these questions, participants then read the specific instructions for the threshold information condition to which they were assigned. The instructions explaining the threshold were identical but differed in two key respects: 1) the level of threshold needed to receive the public good, and 2) the information their group members knew regarding the threshold. The threshold level manipulation had three levels, a low threshold, a high threshold, and a maximum threshold. In the low threshold condition, 2 out of 6 participants in a group needed to contribute their $\$ 0.30$ endowment in order for contributions to be multiplied and split amongst their group members. In the high threshold condition, 4 out of 6 participants in a group needed to contribute. In the maximum threshold condition, all 6 out of 6 participants in a group needed to contribute. Contribution decisions were binary, participants could either contribute the entirety of their $\$ 0.30$ endowment or not contribute. Critically, in our threshold PGG, if participants failed to reach the threshold then all contributions to the common pot were destroyed, leaving them with only the portion of their endowment they did not contribute.

The threshold information manipulation had three conditions: common knowledge, common ignorance, and private knowledge. In the common knowledge condition, participants were told that everyone in their group saw the same 
instructions they did, and that everyone in their group knew that the participant had seen the same instruction as well. Thus, everyone in their group knew that at least 4 out of their 6 group members needed to contribute (or 2 and 6 , in the low and maximum threshold treatments, respectively) or the total common pot would be destroyed. This established something at least broadly consistent with a recursive belief state regarding the threshold such that the participant knew the threshold, knew everyone in their group knew the threshold, and knew that everyone in their group knew that they knew the threshold, ad infinitum. The common ignorance condition was identical to the common knowledge condition but, in this condition, the threshold was unknown (e.g. "the amount you must contribute is unknown to your group"). Thus, the participant did not know the threshold, everyone in their group did not know the threshold, and the participant knew that everyone in their group knew that they did not know the threshold, ad infinitum. We included this condition to investigate whether the presence of a threshold, even when unknown, would be enough to promote contributions in the PGG. In the private knowledge condition, participants were told the threshold level but that they could not be certain that their group members saw the same instructions as them, thus resulting in a lack of common knowledge (e.g. "only you know for certain that at least 4 out of your 6 group members must contribute"). Thus, the participant knew the threshold, but they were not sure if everyone in their groups knew the threshold, and everyone in their group did not know if they knew the threshold. After reading the information threshold instructions, participants answered three more comprehension questions for their specific information and threshold condition. After answering these comprehension questions, participants then made their contribution decision.

Participants could contribute their entire endowment or nothing ( 0 to 30 cents) to their group pot. After making their contribution decision, participants were asked to predict how many other group members would contribute to the public good collectively ( 0 to 5 contributors). We included this question to assess whether participants' predictions of their group members' contributions influenced their own contribution decisions. In order to compare the level of cooperation observed in the information threshold conditions to a standard PGG, we also ran a baseline condition in which participants played an identical PGG without a threshold. We report all measures, manipulations, and exclusions (see supplementary materials for measures not reported here).

Table 1. Table displaying all combinations of threshold level and information conditions. 


\begin{tabular}{|c|c|c|c|}
\hline $\begin{array}{l}\text { Information } \\
\text { Condition }\end{array}$ & Low Threshold & High Threshold & Maximum Threshold \\
\hline $\begin{array}{c}\text { Common } \\
\text { Knowledge }\end{array}$ & $\begin{array}{l}\text { Everyone knows that } \\
\text { everyone knows the } \\
\text { threshold is } 2 \text { out of } 6 \\
\text { people }\end{array}$ & $\begin{array}{l}\text { Everyone knows that } \\
\text { everyone knows the } \\
\text { threshold is } 4 \text { out of } 6 \\
\text { people }\end{array}$ & $\begin{array}{l}\text { Everyone knows that } \\
\text { everyone knows the } \\
\text { threshold is } 6 \text { out of } 6 \\
\text { people }\end{array}$ \\
\hline $\begin{array}{l}\text { Common } \\
\text { Ignorance }\end{array}$ & \multicolumn{3}{|c|}{ Everyone knows that no one knows what the threshold is } \\
\hline $\begin{array}{c}\text { Private } \\
\text { Knowledge }\end{array}$ & $\begin{array}{l}\text { Only I know with } \\
\text { certainty the threshold } \\
\text { is } 2 \text { out of } 6 \text { people }\end{array}$ & $\begin{array}{l}\text { Only I know with } \\
\text { certainty the threshold } \\
\text { is } 4 \text { out of } 6 \text { people }\end{array}$ & $\begin{array}{c}\text { Only I know with } \\
\text { certainty the threshold is } \\
6 \text { out of } 6 \text { people }\end{array}$ \\
\hline Baseline & \multicolumn{3}{|c|}{ No threshold } \\
\hline
\end{tabular}

\subsection{Analyses}

We ran three pre-registered logistic regression models with contribution (binary: 0 = did not contribute, $1=$ did contribute) as the response term. To determine whether information type influenced cooperation, the first model included information (baseline, common knowledge, common ignorance, private knowledge) as the predictor variable (see Information column in Table 3). To determine whether there was an interaction between threshold level and information type, the third model included the interaction between information (private knowledge, common knowledge) and threshold (low, high, max), as well as information condition (private knowledge, common knowledge) and threshold level (low, high, max) as the predictor terms (see the Information $\times$ Threshold column in Table 3). We left out the common ignorance and baseline conditions from this model because threshold level did not vary across baseline or common ignorance conditions, preventing us from examining the interaction between threshold and information. We also ran a third preregistered model predicting contribution decision by threshold level which we report in the Supplement. To determine whether our predictors explained more variance than a null model, we compared the model with the threshold-information condition interaction, plus age and 
gender terms, to a model only including age and gender. The model with the interaction term explained significantly more variance than the model without it $(\chi 2$ $(5)=17.03, p=.004)$.

For all models, we made specific comparisons within information and threshold conditions by using a series of pre-registered pairwise comparisons using estimated marginal means adjusted using the multivariate t method (MVT) to correct for multiple comparisons. These pairwise tests allowed us to make the critical comparison between the common knowledge and private knowledge conditions in order to determine whether common knowledge increased contributions. We next ran two exploratory models that were not preregistered. To test whether participant's predictions about the number of their group members contributing predicted their own contributions, we ran a logistic regression model with contribution as the response term and predicted number of other group members contributing (continuous: 0 -5) as a predictor. We also ran an identical model but included information condition (private knowledge, common knowledge, common ignorance) as a predictor to determine whether it would predict contributions when controlling for predicted group member contributions.

Lastly, in an exploratory model, we examined whether predicted contributors mediated the effect of information condition on contributions by creating a path analysis model with contribution (binary: 0,1 ) as the endogenous variable, information condition (private knowledge, common knowledge) as the exogenous variable, and predicted contributors (continuous: $0-5$ ) as the mediator. We used bootstrapping with 5,000 iterations to find standard errors, bias-corrected bootstrapped confidence intervals with 5,000 samples, and diagonally weighted least squares (DWLS) to estimate the model parameters.

Table 2. Proportions and standard deviations (in parentheses) of contributions in the PGG by information and threshold level.

\begin{tabular}{|c|c|c|c|c|}
\hline & Low & High & Maximum & \\
\hline Baseline & & $0.5(0.5)$ & \\
\hline Common Knowledge & $0.78(0.41)$ & $0.72(0.45)$ & $0.78(0.41)$ & $0.76(0.42)$ \\
\hline Common Ignorance & $0.74(0.44)$ & $0.73(0.45)$ & $0.71(0.45)$ & $0.73(0.45)$ \\
\hline Private Knowledge & $0.70(0.46)$ & $0.72(0.45)$ & $0.65(0.48)$ & $0.69(0.46)$ \\
\hline & $0.74(0.44)$ & $0.72(0.45)$ & $0.71(0.45)$ & \\
\hline
\end{tabular}




\subsection{Results}

\subsubsection{Planned Analyses}

Overall, we found that information level predicted contributions: participants in the common knowledge $(\mathrm{B}=1.15, \mathrm{SE}=.17, \mathrm{p}<.001, \mathrm{OR}: 3.15,95 \% \mathrm{CI}: 2.27,4.37)$, common ignorance $(\mathrm{B}=0.96, \mathrm{SE}=.16, \mathrm{p}<.001, \mathrm{OR}: 2.61,95 \% \mathrm{CI}: 1.89,3.61)$ and private knowledge $(\mathrm{B}=0.76, \mathrm{SE}=.16, \mathrm{p}<.001, \mathrm{OR}: 2.14,95 \% \mathrm{CI}: 1.55,2.96)$ conditions were all significantly more likely to contribute than participants in the baseline condition (see Table 2 for means). Our findings replicate previous work on thresholds, suggesting that people were more likely to contribute to the public good when there was a threshold. Critically, the comparisons between information conditions revealed that participants were significantly more likely to contribute when there was common knowledge of the threshold than when there was private knowledge of the threshold $(\mathrm{B}=-0.39, \mathrm{SE}=.12, \mathrm{p}=.009)$. Participants in the common ignorance condition were not more likely to contribute than participants in the private knowledge condition $(\mathrm{B}=0.19, \mathrm{SE}=.12, \mathrm{p}=.35)$ or those in common knowledge condition $(\mathrm{B}=-0.19, \mathrm{SE}=.12, \mathrm{p}=.42)$.

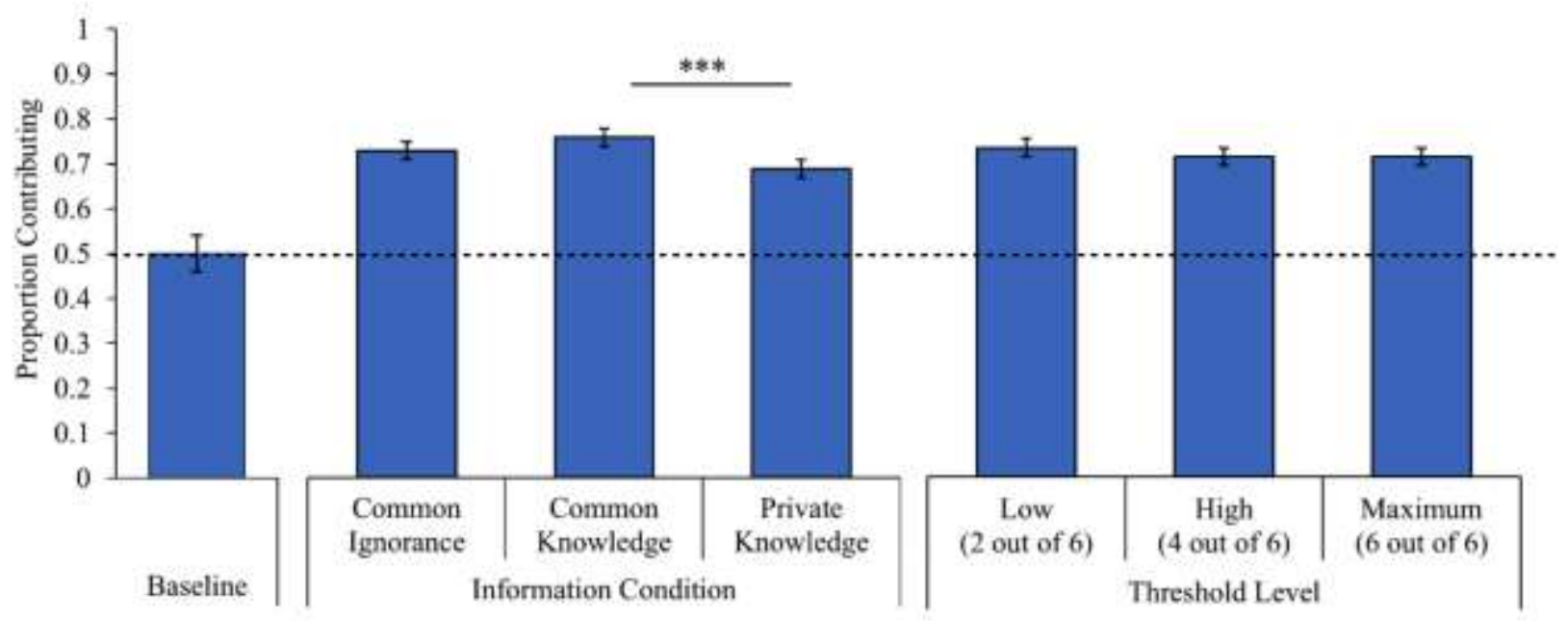

Figure 1. Proportion of participants contributing in the PGG across the three information conditions (common ignorance, common knowledge, private knowledge), the three threshold levels (low: 2 out of 6 , high: 4 out of 6 , maximum: 6 out of 6), and the baseline, non-threshold game. All threshold PGGs elicited more contributions than the baseline condition which is indicated by the dotted line. Error bars indicate standard error. ${ }^{* * *} \mathrm{p}<.001$. 
When predicting contribution by threshold level, information condition, and their interaction, we found that the interaction between threshold (low and high) and information (common knowledge and private knowledge) was not significant (B $=-0.45, \mathrm{SE}=.29, \mathrm{p}=.13, \mathrm{OR}: 0.63,95 \% \mathrm{CI}: 0.35,1.14)$. The interaction between threshold (low and max) and information (private knowledge and common knowledge) was also not significant ( $\mathrm{B}=0.24, \mathrm{SE}=.30, \mathrm{p}=.43, \mathrm{OR}: 1.27,95 \% \mathrm{CI}$ : $0.70,2.29)$. However, the significance of this interaction term hinged on the reference condition for the threshold variable: when we set the reference category to the high condition, the interaction between threshold (high and max) and information (private knowledge and common knowledge) was significant ( $\mathrm{B}=0.69$, $\mathrm{SE}=.29, \mathrm{p}=.02$, OR: 1.99, 95\% CI: 1.11, 3.57). The preregistered pairwise comparisons between the private and common knowledge conditions within each threshold level revealed that within the low threshold condition, participants were significantly more likely to contribute in the common knowledge condition than the private knowledge condition $(\mathrm{B}=-0.46, \mathrm{SE}=.22, \mathrm{p}=.033$ ). Similarly, within the max threshold condition, participants were significantly more likely to contribute in the common knowledge condition than the private knowledge condition $(B=-0.69, \mathrm{SE}=$ $.21, \mathrm{p}=.001$ ). Within the high threshold condition, there was no difference in participants' likelihood of contributing between the common knowledge and the private knowledge conditions $(\mathrm{B}=-0.01, \mathrm{SE}=.21, \mathrm{p}=.98)$.

Next, we found that the more group members participants predicted would contribute, the more likely participants were to contribute themselves $(\mathrm{B}=1.30, \mathrm{SE}$ $=.06, \mathrm{p}<.001$, OR: $3.69,95 \% \mathrm{CI}: 3.27,4.18)$. When controlling for predictions about other group members contributing, information condition ceased to predict contributions; participants were not more likely to contribute when there was common knowledge than private knowledge $(\mathrm{B}=0.19, \mathrm{SE}=.16, \mathrm{p}=.21, \mathrm{OR}: 1.22$, $95 \%$ CI: $0.89,1.66)$ or when there was common ignorance than private knowledge $(\mathrm{B}=0.23, \mathrm{SE}=.15, \mathrm{p}=.13$, OR: $1.26,95 \% \mathrm{CI}: 0.94,1.69)$.

Lastly, we found that the predicted number of group contributors fully mediated the effect of information condition on contributions. The total effect of information condition on contributions was significant $(b=0.23, \mathrm{SE}=0.07, p<.001)$, while the direct effect of information condition on contributions was not significant $(b=.07, \mathrm{SE}=.06, p=.27)$. The path from information condition to predicted contributors was significant $(b=0.325, \mathrm{SE}=.08, p<.001)$, with information condition explaining $11.4 \%$ of the variance in the number of predicted group members contributing (see Table S10 in the Supplement for model output). The path from predicted contributors to PGG contribution ( $b=0.51, \mathrm{SE}=0.01, p<.001$ ) was also significant, with the predicted number of contributors explaining $71.8 \%$ of the variance in contributions in the PGG. Critically, the indirect effect was significant 
( $b=0.16, \mathrm{SE}=0.04, p<.001)$, explaining $8.2 \%$ of the total variance, with the biascorrected bootstrapped confidence interval with 5,000 samples above zero (95\% CI: $0.09,0.24)$.

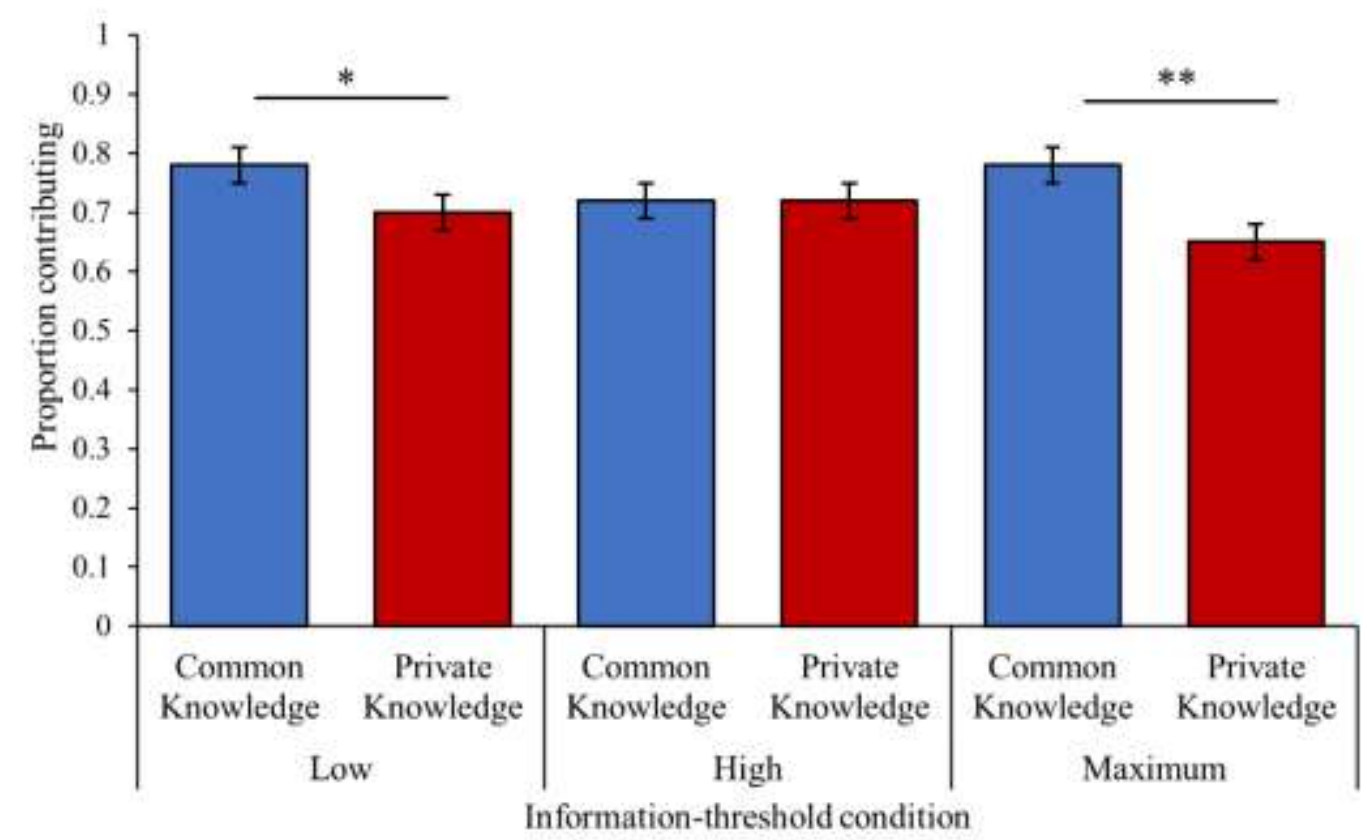

Figure 2. Proportion of participants contributing in the PGG between the common knowledge and private knowledge conditions within the low, high and maximum threshold levels (low: 2 out of 6, high: 4 out of 6 , maximum: 6 out of 6 ). Common knowledge elicited more contributions only within the low and maximum threshold treatments. Error bars indicate standard error. ${ }^{*} \mathrm{p}<.05 ;{ }^{*} \mathrm{p}<.01$.

Table 3. Estimate and standard error of fixed effects in logistic regression models predicting contribution to the public good. The baseline condition was set as the reference category for the information and threshold models. For the information and threshold interaction model, the reference categories were set as follows: Threshold - Low, Knowledge - Common Knowledge. 
In print at Evolution \& Human Behavior

\begin{tabular}{lll} 
(Intercept) & $0.02(0.14)$ & $0.82(0.15)^{* * *}$ \\
Common Ignorance & $0.96(0.17)^{* * *}$ & \\
Private Knowledge & $0.76(0.16)^{* * *}$ & \\
Common Knowledge & $1.15(0.17)^{* * *}$ & $0.46(0.22)^{*}$ \\
High Threshold & & $0.11(0.21)$ \\
Max Threshold & & $-0.22(0.20)$ \\
Low Threshold & & \\
High Threshold $\times$ Common Knowledge & & $-0.45(0.30)$ \\
Max Threshold $\times$ Common Knowledge & & $0.24(0.30)$ \\
\hline AIC & & 1367 \\
BIC & 2684.56 & 1601.93 \\
Log Likelihood & 2707.44 & -794.96 \\
Deviance & & 158.25 \\
\hline & -1338.28 & \\
\hline
\end{tabular}

${ }^{* * * *} \mathrm{p}<0.001,{ }^{* *} \mathrm{p}<0.01,{ }^{*} \mathrm{p}<0.05$

\subsection{Discussion}

Consistent with our predictions, we found that participants contributed more to the public good when there was common knowledge of the threshold compared to when there was only private knowledge. We also found tentative evidence that the effect of common knowledge was at least partly due to increased certainty in the number of group members contributing. Additionally, we replicated past work showing that thresholds increase cooperation in the public goods game; contributions were higher across all threshold levels relative to the baseline condition that lacked a threshold (Jordan et al., 2017; Szolnoki \& Perc, 2010; van de Kragt et al., 1983). When we examined the interaction between threshold level and 
information condition, we found that participants contributed significantly more when there was common knowledge, but only in the low and maximum threshold conditions. This potentially suggests that the effect of common knowledge on cooperation depends on the threshold level. However, because we did not predict an interaction between common knowledge and threshold at the outset, we are reluctant to further interpret this finding. We attempt to replicate this interaction in Study 2 to better understand whether the effect of common knowledge does in fact hinge on the threshold level.

A key finding from this study was that the effect of common knowledge on contributions might have resulted from increased certainty regarding whether group members would contribute. Specifically, we found that the expected number of group members contributing predicted participant's own contribution decisions and when controlling for predictions about how many group members would contribute to the public good, information condition no longer predicted contributions. Furthermore, a mediation analysis revealed that the predicted number of contributors fully mediated the effect of information condition on contributions. These findings provide initial evidence that the increased number of contributions in the common knowledge condition may have been a result of decreased uncertainty about group member's cooperative behavior. These results support our prediction that certainty serves as a mechanism underlying the effectiveness of common knowledge on coordination. In Study 2, our aim was, first, to extend our findings from Study 1 by explicitly investigating whether the effect of common knowledge on coordination is mediated by certainty about group members' cooperative behavior, and second, to replicate our findings from Study 1.

\section{Study 2}

\subsection{Participants}

We tested $\mathrm{N}=1859$ participants $(56.7 \%$ female $)$, aged 18-91 ( $\mathrm{M}=40.7)$ from Amazon's Mechanical Turk in a preregistered study (https://osf.io/brqky/?view_only=ab7c7982f7454e439ca63c5806c00d52). This sample size was based on that used in previous work in threshold PGGs conducted on Mechanical Turk (Jordan et al., 2017). While we initially recruited 2,866 participants, we excluded 447 from analysis for failing to complete the study in its entirety, 527 for failing the comprehension questions, and 18 for responding with "three or more" to a question assessing the number of questions they answered without reading or thinking about them carefully. Additionally, 15 were excluded for completing the survey more than once (we included first responses) or for 
completing the survey after an incomplete attempt in which they were exposed to an experimental condition.

\subsection{Design \& Procedure}

In Study 2, we focused on a subset of the most interesting threshold and information conditions. To replicate the effect of common knowledge on cooperation, we included the two information conditions in which common knowledge of the threshold was present (common knowledge) or absent (private knowledge). Additionally, because the effect of common knowledge differed by threshold level in Study 1, we included a threshold level in which the effect was the strongest (maximum) and one in which it was entirely absent (high). To capture the baseline level of cooperation, we again included a condition without a threshold. In total, participants were assigned to one of five conditions.

The procedure of Study 2 was identical to Study 1 in every respect (i.e. the game and threshold information manipulations were the same) with the exception of the following differences. After participants read the PGG and threshold information instructions and answered the comprehension questions, they either first made their PGG contribution decision followed by their prediction about the number of group members contributing, or first made their prediction about the number of contributors followed by their PGG contribution. The order of dependent variables was counterbalanced to control for order effects. Unlike Study 1, the question assessing participants' predicted number of contributors was incentivized, such that participants received $\$ 0.10$ for correctly predicting the number of their group members who contributed to the public good. Directly after answering this prediction question, participants answered a question assessing their certainty about their response (0-100 sliding scale, anchored from 0-"not certain at all" to 100-"extremely certain"). Previous work has used a similar self-report approach to measure certainty and confidence (Balakrishnan \& Ratcliff, 1996; Bradley, 1981; Thunström et al., 2015). After completing both blocks of dependent variables, participants answered an exploratory question that elicited individual-level certainty distributions for the number of their group members contributing.

\subsection{Analyses}

To replicate our analysis from Study 1, we ran three pre-registered logistic regression models with contribution (binary: $0=$ did not contribute, $1=$ did contribute) as the response term. To determine whether information condition influenced contributions, the first model included information (baseline, private knowledge, common knowledge) as the predictor variable (see the Information column in Table 4). To determine whether there was an interaction between 
information condition and threshold level, the third model included information (private knowledge, common knowledge), threshold (high, maximum), and the interaction between information and threshold (see the Information $\times$ Threshold column in Table 4). We also ran a third preregistered model predicting contributions by threshold which we report in the Supplement. For all three models we made comparisons within information and threshold condition with a series of preregistered pairwise comparisons using estimated marginal means adjusted using the multivariate $t$ method (MVT) to correct for multiple comparisons. For all models, we dummy coded the information condition and threshold level categorical predictors, setting private knowledge and high threshold conditions as the reference categories, respectively. As in Study 1, we compared the model with the interaction term, in addition to gender and age, to a null model without the interaction term. The model with the interaction term explained significantly more variance in contributions than the null model $(\chi 2(3)=9.19, p=.027)$.

To determine whether the distributions of predicted contributors and certainty ratings differed between the private and common knowledge conditions, we ran two, preregistered two-sample bootstrap Kolmogorov-Smirnov (KS) tests. Deviating from our pre-registration, we used the ks.boot command in the "Matching" package (Sekhon, 2011) to run bootstrapped KS tests with 5000 iterations in order to handle the ties in our data. We next ran another set of five pre-registered logistic and linear regression models to assess the relationship between information condition (private knowledge, common knowledge), threshold level (high, maximum), predicted contributors (continuous: $0-5$ ), certainty scores (continuous: 0-100), dependent variable order (PGG contribution, PGG contribution prediction), and PGG contributions (binary: $0=$ did not contribute, $1=$ did contribute). We report the results of these models in the SOM but note here that they conform with our predictions indicating there was no order effect of dependent variable.

To examine whether the participants' predictions about the number of contributors and their certainty about those predictions mediated the effect of common knowledge on contributions, we ran two, pre-registered structural equation models to test for indirect effects. To determine whether predicted contributors mediated the effect of information condition on contributions, we created a path analysis model with contribution (binary: 0,1 ) as the endogenous variable, information condition (private knowledge, common knowledge) as the exogenous variable, and predicted contributors (continuous: $0-5$ ) as the mediator. To determine whether certainty ratings for predicted contributors mediated the effect of information condition on contributions, we created a path analysis model with contribution (binary: 0,1 ) as the endogenous variable, information condition (private knowledge, common knowledge) as the exogenous variable, and certainty ratings (continuous: 0-100) as the mediator. For both models, we used 
bootstrapping with 5,000 iterations to find standard errors, bias-corrected bootstrapped confidence intervals with 5,000 samples, and diagonally weighted least squares (DWLS) to estimate the model parameters.

Table 4. Proportions and standard deviations (in parentheses) of contributions in the PGG by information and threshold level.

\begin{tabular}{|c|c|c|c|}
\hline & High & Maximum & \\
\hline Baseline & \multicolumn{2}{|c|}{$0.66(0.48)$} & \\
\hline Common Knowledge & $0.78(0.41)$ & $0.81(0.39)$ & $0.80(0.40)$ \\
\hline Private Knowledge & $0.72(0.45)$ & $0.76(0.43)$ & $0.74(0.44)$ \\
\hline & $0.75(0.43)$ & $0.78(0.41)$ & \\
\hline
\end{tabular}

\subsection{Results}

\subsubsection{Replication of Study 1 Results}

Replicating results from Study 1, we found that information condition predicted contributions: participants were more likely to contribute in the private knowledge $(\mathrm{B}=0.38, \mathrm{SE}=0.14, p=.006$, OR: $1.46,95 \% \mathrm{CI}: 1.55,2.71)$ and common knowledge conditions ( $\mathrm{B}=0.72$, SE $=0.14, p<.001$, OR: $2.05,95 \% \mathrm{CI}: 1.55,2.71$ ) than in the baseline condition (see Table 4 for means). Critically, the within information condition comparison found that participants were significantly more likely to contribute when there was common knowledge than when there was private knowledge $(\mathrm{B}=0.34, \mathrm{SE}=0.12, p=.017)$.

When predicting contributions by threshold level, information condition, and their interaction, we found that participants were significantly more likely to contribute when there was common knowledge than private knowledge $(\mathrm{B}=0.35$, $\mathrm{SE}=0.17, p=.038$, OR: $1.42,95 \% \mathrm{CI}: 1.02,1.98)$. Participants were not more likely to contribute when the threshold was maximum than high $(\mathrm{B}=0.19, \mathrm{SE}=0.17, p=$ .263, OR: 1.21, 95\% CI: 0.87, 1.68). The interaction between information condition and threshold level was also not significant $(\mathrm{B}=-0.04, \mathrm{SE}=0.25, p=.86,0 \mathrm{R}: 0.96$, $95 \% \mathrm{CI}: 0.59,1.55)$, suggesting that the difference between information condition did not vary by threshold level. The pairwise comparisons revealed that within the high threshold level, participants were significantly more likely to contribute in the common knowledge than private knowledge condition $(\mathrm{B}=0.35, \mathrm{SE}=0.17, p=$ .038). Within the maximum threshold level, the difference in contributions between when there was common knowledge compared to private knowledge was trending on significance $(\mathrm{B}=0.31, \mathrm{SE}=0.18, p=.08)$. 


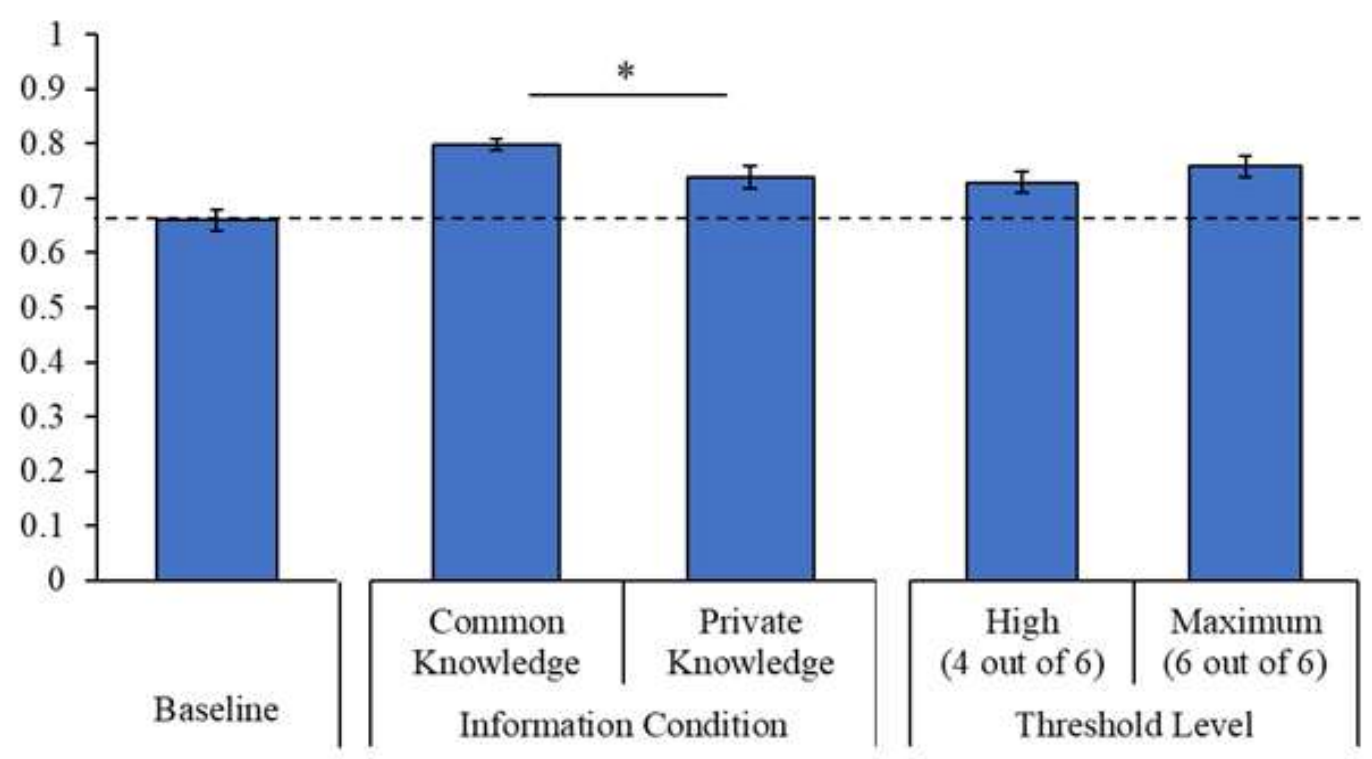

Figure 3. Proportion of participants contributing in the PGG across the two information conditions (common knowledge, private knowledge), the two threshold levels (high: 4 out of 6, maximum: 6 out of 6), and the baseline, non-threshold game. All threshold PGGs elicited more contributions than the baseline condition, which is indicated by the dotted line. Error bars indicate standard error. ${ }^{*} \mathrm{p}<.05$.

Table 5. Estimate and standard error of fixed effects in logistic regression models predicting contribution to the public good. The baseline condition was set as the reference category for the information and threshold models. For the information and threshold interaction model, the reference categories were set as follows: Threshold - Low, Knowledge - Common Knowledge.

\section{Information Information $\times$ Threshold}

$\begin{array}{lll}\text { (Intercept) } & 0.64(0.11)^{* * *} & 0.94(0.11)^{* * *} \\ \text { Common Knowledge } & 0.72(0.14)^{* * *} & 0.35(0.17)^{*} \\ \text { Private Knowledge } & 0.38(0.14)^{* *} & \end{array}$

Max Threshold

High Threshold 
In print at Evolution \& Human Behavior

Max Threshold $\times$ Common

$-0.04(0.25)$

Knowledge

\begin{tabular}{lll}
\hline AIC & 2091.22 & 1623.84 \\
BIC & 2107.80 & 1645.08 \\
Log Likelihood & -1042.61 & -807.92 \\
Deviance & 2085.22 & 1615.84 \\
Num. obs. & 1859 & 1496 \\
\hline
\end{tabular}

\subsubsection{Additional analyses}

The distributions of predicted contributors differed significantly between the common knowledge and private knowledge conditions $(\mathrm{D}(1859)=0.15, p<.001)$. A qualitative appraisal of the distributions suggests that the common knowledge distribution was skewed towards a higher number of predicted contributors than the private knowledge distribution (see Supplement for histograms of predicted contributors and certainty ratings). When examining whether the distribution of certainty ratings differed between common knowledge and private knowledge conditions, we found that the distributions differed significantly $(D(1859)=0.13, p<$ .001). Again, a qualitative examination of the distributions suggests that the common knowledge distribution was skewed towards higher certainty ratings than the private knowledge condition.

\subsubsection{Mediation}

We found that the predicted number of group contributors fully mediated the effect of information condition on contributions (see Figure 4 for a path diagram). The total effect of information condition on contributions was significant $(b=0.196$, $\mathrm{SE}=0.07, p=.007)$, while the direct effect of information condition on contributions was not significant ( $b=.038, \mathrm{SE}=.055, p=.487)$. The path from information condition to predicted contributors $(b=0.30, \mathrm{SE}=.07, p<.001)$ was significant, with information condition explaining $10.9 \%$ of the variance in the number of predicted group members contributing (see Table S11 in the Supplement for model output). The path from predicted contributors to PGG contribution ( $b=0.52$, SE $=$ $.016, p<.001$ ) was also significant, with the predicted number of contributors explaining $72.5 \%$ of the variance in contributions in the PGG. Critically, the indirect effect was significant ( $b=-0.16, \mathrm{SE}=0.04, p<.001$ ), explaining $7.8 \%$ of the total 
variance, with the bias-corrected bootstrapped confidence interval with 5,000 samples above zero (95\% CI: 0.07, 0.23).

Next, we found that certainty about the number of predicted contributors fully mediated the effect of information condition on contributions (see Figure 5 for a path diagram). The total effect of information condition on contributions was again significant $(b=.196, \mathrm{SE}=0.07, p=.006$ ) but the direct effect of information condition on contributions was not significant $(b=0.08, \mathrm{SE}=0.07, p=.242)$. The path from information condition to certainty ratings $(b=8.62, \mathrm{SE}=1.37, p<.001)$ was significant, with information condition explaining $16.2 \%$ of the variance in certainty ratings (see Table S12 in the Supplement for model output). The path from certainty ratings to PGG contributions ( $b=0.013, \mathrm{SE}=.001, p<.001)$ was also significant, with certainty explaining $34.5 \%$ of the variance in contributions to the public good. Importantly, the indirect effect was significant $(b=0.11, \mathrm{SE}=.02, p<$ .001 ), explaining $5.6 \%$ of the total variance, with the bias-correct bootstrapped confidence interval with 5,000 samples above zero (95\% CI: 0.08, 0.16). As preregistered, we replicate the results of these mediation analyses using a different approach (see supplement). We note here that the results do not differ in interpretation.

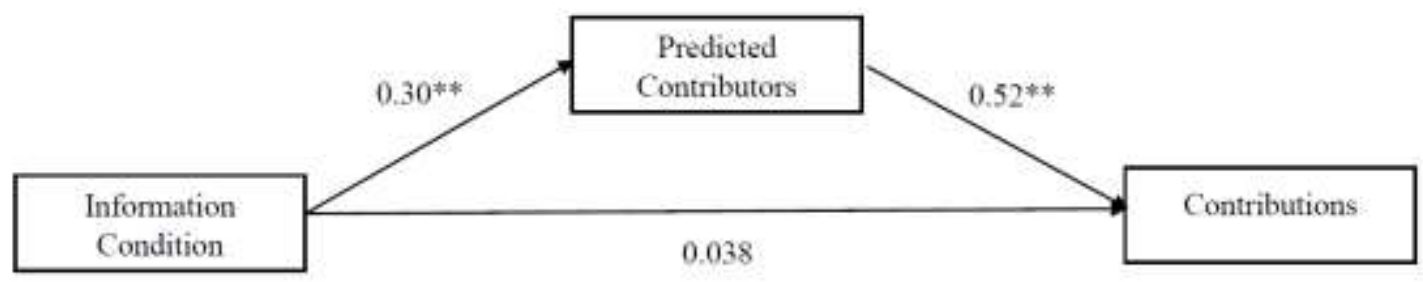

Figure 4. Diagram of the path analysis model with predicted contributor as a mediator. ${ }^{* *} \mathrm{p}<.01$.

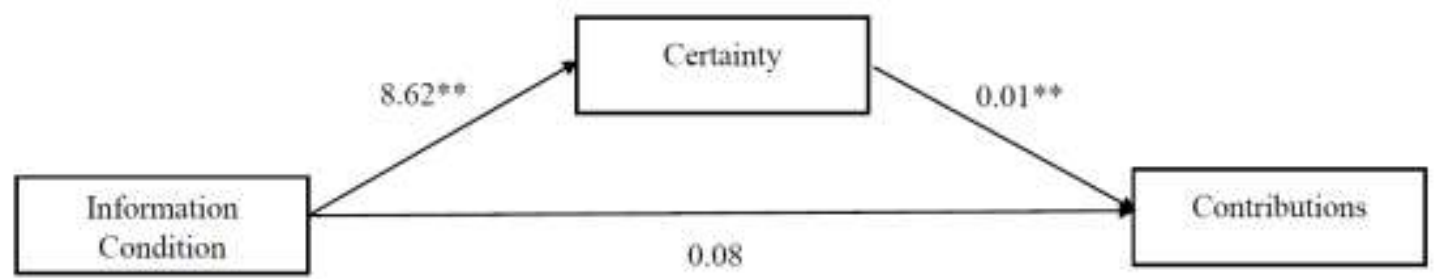

Figure 5. Diagram of the path analysis model with certainty as a mediator. ${ }^{* *} \mathrm{p}<.01$.

\subsection{Discussion}


The central aim of Study 2 was to test the mediating role of certainty and predicted group contributors on PGG contributions. Results of our path analyses provide compelling evidence that the effect of common knowledge on increased contributions was mediated by the predicted number of group members contributing and certainty ratings about these predictions. Additionally, the KS tests suggested that the distributions of predicted contributors and certainty ratings were significantly different and were more negatively skewed in the common knowledge condition compared to the private knowledge condition. This suggests that when there was common knowledge, participants were more likely to think that more of their group members would contribute and were more certain of those predictions. Overall, the results of these analyses provide strong evidence that common knowledge increases contributions by decreasing uncertainty about group members' cooperative behavior.

In Study 2, we also sought to replicate and extend our findings from Study 1. Just as in Study 1, we found an effect of information condition on contributions: participants were more likely to contribute when there was common knowledge compared to private knowledge. However, unlike Study 1, we did not see an interaction between threshold level and information condition: there was not a larger effect of common knowledge on contributions within the maximum threshold level compared to the high threshold level. In fact, the effect of common knowledge was actually stronger in the high than the maximum threshold games. Overall, our results suggest that threshold level is not a strong determinant of the effect of common knowledge on cooperation. Interestingly, we observed much higher baseline levels of cooperation, and a relatively smaller effect of common knowledge, in Study 2 than Study 1, perhaps reflecting post-COVID changes to the Mechanical Turk participant pool (Arechar \& Rand, preprint).

One as yet unaddressed question is why we observed intermediate levels of contributions in the common ignorance condition in Study 1 . That is, even when participants did not know the threshold level, and knew that their group did not either, they still contributed at levels in-between those observed in the common and private knowledge conditions. On its face, this result could be construed as problematic for our account-if uncertainty mediates contribution decisions, why are people contributing in the common ignorance condition at all? However, participants in this condition still possessed common knowledge that there was $a$ threshold, and in the absence of threshold level information, participants might have simply assumed that there was a threshold of intermediate size. Furthermore, because there is common knowledge that there is a threshold, we expect that certainty in others' contributions underlies cooperation in this condition, much like it does in the common knowledge condition: Participants who predict that more of their group members will contribute, and are more certain in those predictions, will 
be more likely to contribute, even if there is uncertainty about the specific threshold level. In Study 3, we sought to to replicate the mediating role of certainty on cooperation and more conclusively determine whether the effect of common knowledge varies by threshold size. Additionally, we aimed to explain the contribution levels observed in the common ignorance condition in Study 1, and test our prediction that uncertainty about others' cooperative behavior is the mechanism that underlies contributions. We also introduced a new common ignorance condition in which there is uncertainty regarding whether there is a threshold at all (and if there is, what level it is) to explore whether the certainty about the presence of a threshold might explain the contribution levels observed in the common ignorance condition from Study 1.

\section{Study 3}

\subsection{Participants}

We tested $\mathrm{N}=1469$ participants $(58.82 \%$ female $)$, aged $19-83(\mathrm{M}=40.02)$ on Amazon's Mechanical Turk in a preregistered study (https://osf.io/xqyip/?view only=7605805b183e470daafc2d7d0a535c88). This sample was based on previous work in the threshold PGGs conducted on Mechanical Turk (Jordan et al., 2016) and a power analysis in $G^{*}$ Power which suggested we'd have $98 \%$ power to detect a small odds ratio for the effect of common knowledge on contributions. We initially recruited 2,509 participants, 346 were excluded for failing to complete the entire study, 681 for failing any of the comprehension questions, and 13 for responding with "three or more" to a question assessing the number of questions they answered without reading or thinking about them carefully. The exclusion rate is higher than in previous studies, partly due to a programming error with a comprehension check in the survey in one of the common ignorance conditions that impacted about half of participants in this condition (see SOM for details). We include these participants in our data set and note that contributions, predicted contributors, and certainty ratings did not differ between impacted and unimpacted participants.

\subsection{Design \& Procedure}

This Study included the same information (common knowledge, private knowledge) and threshold level (low, high) conditions as in Study 2, but with the addition of two common ignorance conditions. To determine whether certainty ratings also mediate contributions in the common ignorance condition, we added the same common ignorance condition from Study 1, but this time including our certainty measures from Study 2. Additionally, we included a new common 
ignorance condition in which there was uncertainty regarding whether there was a threshold or not, and if there was, its size. Thus, participants were assigned to one of six conditions between-subjects.

The procedure of Study 3 was identical to Study 2 in every respect with the exception that participants in the common ignorance conditions made a threshold level prediction, in which they were asked to predict the size of the threshold (0-6), after answering their predicted contributor and certainty ratings. As in Study 2, participants read the game instructions, answered the comprehension questions, and then made their PGG contribution and PGG predicted contribution and certainty decisions in a counterbalanced order.

\subsection{Analyses}

To replicate our results from Study 2, we ran four pre-registered analyses. First, to replicate our finding that people are more likely to contribute when there is common knowledge, we ran a logistic regression with contribution (binary: $0=$ did not contribute, 1 = did contribute) as the response term and information condition (common knowledge, common ignorance, private knowledge) as a predictor. Second, to determine whether the effect of common knowledge varies across threshold levels, we ran another logistic regression with contribution as the response term and information condition, threshold size (high, max), and their interaction as predictors. Lastly, to replicate the mediation models showing that predicted contributors and certainty ratings mediated the effect of information condition on contributions, we ran two path analysis mediation models. The mediation models included contribution as the endogenous variable, information condition (private knowledge, common knowledge) as the exogenous variable and predicted contributors (continuous: $0-5$ ) and certainty (continuous: 0-100) as mediators. For both models, and all subsequent mediation models, we used bootstrapping with 5,000 iterations to find standard errors, bias-corrected bootstrapped confidence intervals with 5,000 samples, and diagonally weighted least squares (DWLS) to estimate the model parameters.

We next ran seven pre-registered models unique to Study 3. To examine whether people are most likely to contribute when there is common knowledge of the threshold as compared to common ignorance of the threshold size, common ignorance of a threshold, or private knowledge, we ran a logistic regression with contribution as the response term and information condition as the predictor. We also examined whether the predicted number of contributors and certainty in those predictions within the two common ignorance conditions predict contributions with four logistic regression models. Two models predicted contribution by predicted contributors, one with the common ignorance-old data and another with just the common ignorance-new data, while the other two models predicted contribution by 
certainty ratings, one with the common ignorance-old data, and another with the common ignorance-new data. We ran a series of bootstrap KS tests to compare the distribution of predicted contributors and certainty ratings between the common ignorance conditions and the common knowledge and private knowledge conditions. To determine whether expected threshold level in the common ignorance conditions predicted contributions, predicted contributors, and certainty ratings, we ran six logistic regression models, three with the common ignorance-old data and three with the common ignorance-new data, including contributions, predicted contributors, and certainty ratings as the response terms.

To determine whether participants are more likely to contribute and predicted a higher threshold level when there is common ignorance of the threshold size (common ignorance-old condition) as compared to when there is common ignorance of whether there is a threshold or not (and if so, what the threshold is; common ignorance-new condition) we ran two logistic regression model with common ignorance condition (common ignorance-old, common ignorance-new) as the predictor and either contribution or expected threshold level as the response term. To explore whether predicted contributors and certainty ratings mediated the difference between the common ignorance conditions on contributions, we ran two mediation path analysis models. These models included common ignorance condition (common ignorance-old, common ignorance-new) as the exogenous variable and either predicted contributors (continuous: $0-5$ ) or certainty (continuous: 0-100) as mediators.

Table 6. Proportions and standard deviations (in parentheses) of contributions in the PGG by information and threshold level.

\begin{tabular}{|c|c|c|c|}
\hline & High & Maximum & \\
\hline Common Knowledge & $0.82(0.39)$ & $0.85(0.36)$ & $0.83(.37)$ \\
\hline Common Ignorance-New & & $0.75(0.44)$ & \\
\hline Common Ignorance-Old & & $0.77(0.42)$ & \\
\hline Private Knowledge & $0.77(0.42)$ & $0.79(0.41)$ & $0.78(0.42)$ \\
\hline & $0.79(0.40)$ & $0.82(0.38)$ & \\
\hline
\end{tabular}

\subsection{Results}

\subsubsection{Replication of Study 2}

Replicating the results from Study 2, we found that participants were more likely to contribute when there was common knowledge than when there was 
private knowledge of the threshold $(\mathrm{B}=-0.36, \mathrm{SE}=0.16, p=.02, \mathrm{OR}: 0.69,95 \% \mathrm{CI}$ : $0.51,0.95)$. Participants were significantly more likely to contribute when there was common knowledge than common ignorance $(\mathrm{B}=-0.43, \mathrm{SE}=0.20, p=.03,0 \mathrm{R}: 0.65$, $95 \%$ CI: 0.44, 0.97). A planned pairwise comparison revealed that participants were as likely to contribute when there was private knowledge as common ignorance (B $=0.07, \mathrm{SE}=0.19, p=.93$ ). When predicting contributions by threshold level, information condition, and their interaction, we found that participants were no longer more likely to contribute when there was common knowledge than private knowledge $(\mathrm{B}=-0.31, \mathrm{SE}=0.22, p=.15$, OR: 0.74, 95\% CI: 0.48, 1.12). The interaction between information condition and threshold level was also not significant (B = -0.12, $\mathrm{SE}=0.32, p=.71, \mathrm{OR}: 0.89,95 \% \mathrm{CI}: 0.47,1.66)$, replicating the null effect found in Study 2. Planned pairwise comparisons revealed that within the high threshold level, participants were not more likely to contribute when there was common knowledge than private knowledge $(\mathrm{B}=0.31$, $\mathrm{SE}=0.22, p=.15)$. Within the maximum threshold level, participants were not significantly more likely to contribute when there was common knowledge compared to private knowledge, although the effect was trending on significance ( $\mathrm{B}=0.43, \mathrm{SE}=0.24, p=.07$ ).

Replicating the previous mediation effects of predicted contributors found in Study 2, we found that the predicted number of group contributors fully mediated the effect of information condition on contributions. The total effect of information condition on contributions was significant ( $b=0.20, \mathrm{SE}=0.09, p=.02$ ), while the direct effect of information condition on contributions was not significant $(b=.04$, $\mathrm{SE}=.07, p=.58$ ). The path from information condition to predicted contributors $(b$ $=0.30, \mathrm{SE}=.08, p<.001$ ) was significant, with information condition explaining $11.5 \%$ of the variance in the number of predicted group members contributing (see Table S13 in the Supplement for model output). The path from predicted contributors to PGG contribution $(b=0.55$, SE $=.02, p<.001)$ was also significant, with the predicted number of contributors explaining $72 \%$ of the variance in contributions in the PGG. Critically, the indirect effect was significant $(b=0.17, \mathrm{SE}=$ $0.05, p<.001$ ), explaining $8.3 \%$ of the total variance, with the bias-corrected bootstrapped confidence interval with 5,000 samples above zero ( $95 \% \mathrm{CI}$ : 0, 0.).

We also replicated the effect of participants' certainty on contributions from Study 2: our mediation model again found that the number of predicted contributors fully mediated the effect of information condition on contributions. The total effect of information condition on contributions was again significant $(b=0.11$, $\mathrm{SE}=0.02, p<.001$ ) but the direct effect of information condition on contributions was not significant ( $\mathrm{b}=0.09, \mathrm{SE}=0.09, p=.29$ ). The path from information condition to certainty ratings $(\mathrm{b}=10.14, \mathrm{SE}=1.62, p<.001)$ was significant, with information condition explaining $19.1 \%$ of the variance in certainty ratings (see Table S14 in the Supplement for model output). The path from certainty ratings to 
PGG contributions $(\mathrm{b}=0.01, \mathrm{SE}=.002, p<.001$ ) was also significant, with certainty explaining $28.4 \%$ of the variance in contributions to the public good. Importantly, the indirect effect was significant ( $\mathrm{b}=0.11, \mathrm{SE}=.02, p<.001)$, explaining $5.4 \%$ of the total variance, with the bias-correct bootstrapped confidence interval with 5,000 samples above zero (95\% CI: 0.07, 0.16).

\subsubsection{Additional Analyses}

Participants were more likely to contribute when there was common knowledge of the threshold than when there was private knowledge $(B=-0.36, \mathrm{SE}=$ $0.16, p=.02$, OR: $0.69,95 \% \mathrm{CI}: 0.51,0.95)$, common ignorance of the threshold size $(B=-0.43, S E=0.20, p=.03$, OR: $0.65,95 \% \mathrm{CI}: 0.44,0.97)$, or common ignorance of the presence of a threshold $(\mathrm{B}=-0.54, \mathrm{SE}=0.19, p=.004, \mathrm{OR}: 0.58,95 \% \mathrm{CI}: 0.40$, 0.85). In the common ignorance-old condition, in which the threshold size was unknown, participants' predicted number of group contributors $(\mathrm{B}=1.36, \mathrm{SE}=0.22$, $p<.001$, OR: $3.88,95 \% \mathrm{CI}: 2.63,6.15)$ and certainty in those predictions (B $=0.02, \mathrm{SE}$ $=0.006, p=.002$, OR: $1.02,95 \%$ CI: $1.01,1.03$ ) significantly predicted contributions. We find the same pattern for the common ignorance-new condition in which the presence of a threshold was unknown: predicted number of group contributors $(\mathrm{B}=$ $1.00, \mathrm{SE}=0.15, p<.001$, OR: $2.73,95 \% \mathrm{CI}: 2.08,3.71)$ and certainty in those predictions $(\mathrm{B}=0.02, \mathrm{SE}=0.006, p<.001$, OR: 1.02, 95\% CI: 1.01, 1.03) both significantly predicted contributions.

When comparing the distributions of predicted contributors, we found that the distributions differed significantly between the common knowledge and common ignorance-old conditions $(\mathrm{D}(734)=0.23, p<.001)$. A qualitative appraisal of the distributions shows that the common knowledge distribution skewed towards a greater number of expected contributors (see Supplement for histograms of predicted contributors and certainty ratings). The distributions of predicted contributors between private knowledge and common ignorance-old conditions was significant $(\mathrm{D}(699)=0.09, p=.047)$ : the common ignorance-old distribution was considerably more uniform than the left skewing distribution in the private knowledge condition. The common ignorance-new and common ignorance-old $(\mathrm{D}(438)=0.08, p=.15)$ did not differ significantly.

Comparing the distribution of certainty in predicted contributors, we found that the distribution of certainty ratings differed significantly between the common knowledge and common ignorance-old conditions $(\mathrm{D}(734)=0.15, p=.001)$. A qualitative appraisal here suggests that the common knowledge distribution was skewed towards higher certainty ratings than the common ignorance-old condition. The certainty distributions did not differ significantly between the private knowledge and common ignorance-old conditions $(\mathrm{D}(699)=0.05, p=.81)$ or 
between the common ignorance-old and common ignorance-new conditions $(\mathrm{D}(438)=0.04, p=0.97)$.

Predicted threshold size within the common ignorance-old did not significantly predict PGG contributions $(\mathrm{B}=0.004, \mathrm{SE}=0.14, p=.98,0 \mathrm{R}: 1.00,95 \%$ CI: $0.76,1.34)$. Predicted threshold size significantly predicted the expected number of group members contributing $(\mathrm{B}=0.21, \mathrm{SE}=0.08, p=.006, \mathrm{OR}: 1.24,95 \% \mathrm{CI}: 1.06$, 1.44 ) but it did not predict certainty ratings in those predictions $(\mathrm{B}=2.24, \mathrm{SE}=1.66$, $p=.18$, OR: 9.43, 95\% CI: 0.36, 248.2). When looking at these same models within the common ignorance-new condition, we find that the predicted threshold size predicted contributions ( $\mathrm{B}=0.22, \mathrm{SE}=0.09, p=.01, \mathrm{OR}: 1.25,95 \% \mathrm{CI}: 1.05,1.50$ ) and the number of predicted group contributors $(\mathrm{B}=0.13, \mathrm{SE}=0.05, p=.02$, OR: $1.13,95 \%$ CI: $1.02,1.26)$ but did not significantly predict certainty in those predictions ( $\mathrm{B}=1.56, \mathrm{SE}=1.05, p=.14$, OR: 4.74, 95\% CI: 0.59, 37.67). Participants were no more likely to contribute to the public good when there was common ignorance of the threshold size as compared to common ignorance of the presence of a threshold ( $\mathrm{B}=-0.11, \mathrm{SE}=0.22, p=.64$, OR: $0.90,95 \% \mathrm{CI}: 0.58,1.39$ ), nor were they more likely to predict a higher threshold size between common ignorance conditions ( $\mathrm{B}=-0.03, \mathrm{SE}=0.14, p=.86$, OR: $0.97,95 \% \mathrm{CI}: 0.74,1.28$ ).

When exploring whether predicted contributors mediates the effect of common ignorance condition (old vs. new) on contributions, we found that neither the total effect of common ignorance condition on contributions $(b=-0.06, \mathrm{SE}=$ $0.13, p=.64$ ), nor the direct effect were significant ( $\mathrm{b}=-0.08, \mathrm{SE}=0.11, p=.46)$. The path from common ignorance condition to predicted contributors was not significant $(\mathrm{b}=0.04, \mathrm{SE}=0.13, p=.75)$, with common ignorance condition explaining $1.5 \%$ of the variance in the number of predicted contributors (see Table S15 in the Supplement for model output). The path from predicted contributors to contributions was significant $(\mathrm{b}=0.48, \mathrm{SE}=0.03, p<.001)$, with predicted contributors explaining $64.5 \%$ of the variance in contributions. Critically, the indirect effect was not significant ( $b=0.02, \mathrm{SE}=0.06, p=.75)$, explaining $1.0 \%$ of the variance, with bias-corrected bootstrapped confidence intervals with 5,000 samples spanning zero (95\% CI: $-0.10,0.14)$.

When exploring whether certainty of predicted contributors mediates the effect of common ignorance condition on contributions, we found that the total effect of common ignorance condition on contributions ( $\mathrm{b}=-0.06, \mathrm{SE}=0.13, p=.99$ ) and the direct effect $(b=-0.06, \mathrm{SE}=0.13, p=.63$ ) were not significant. The path from common ignorance condition to certainty ratings was also not significant $(\mathrm{b}=$ 0.02 , $\mathrm{SE}=2.61, p=.99$ ), with common ignorance condition explaining $0 \%$ of the variance in certainty (see Table S16 in the Supplement for model output). The path from certainty ratings to contributions was significant $(\mathrm{b}=0.01, \mathrm{SE}=0.002, p<$ .001 ), with certainty explaining $30.1 \%$ of the variance in contributions. Critically, 
the indirect effect was not significant $(b=0.00, \mathrm{SE}=0.03, p=.99)$, explaining $0 \%$ of the variance, with bias-corrected bootstrapped confidence intervals with 5,000 samples spanning zero (95\% CI: $-0.06,0.06)$.

\subsection{Discussion}

In line with our predictions, we found that participants' predictions of the number of group members contributing and their certainty in those predictions predicted contributions when there was common ignorance of the threshold. In other words, when participants had common knowledge that there was a threshold of unknown size, certainty about other group members' cooperative behavior supported the effect of common knowledge on contributions. Furthermore, we found that, in the absence of a specific threshold level, participants inferred that the threshold was at an intermediate level. However, in contrast to Study 1, we found that participants were significantly more likely to contribute when there was common knowledge than private knowledge or common ignorance, a finding that suggests a unique effect of common knowledge of the threshold size. In line with Study 1, participants were as likely to contribute when there was common ignorance as private knowledge. Thus, the fact that contributions, and the number of expected contributors, in the common ignorance condition were not significantly different from private knowledge is likely due to participants inferring an intermediate threshold level and were guided by their certainty that their group members knew there was a threshold. While we initially predicted that participants would be more likely to contribute when there was common ignorance of the threshold size compared to common ignorance of whether there was a threshold at all, we failed to find differences in cooperation between these two conditions. However, we believe this is due to the fact that very few participants in the common ignorance-new condition thought there was no threshold: participants made similar predictions about the threshold size between conditions, suggesting they treated these conditions very similarly. Indeed, participants made nearly identical predictions about the number of contributors and certainty ratings between the common ignorance conditions, results which likely explain why we failed to find to evidence of mediation from the common ignorance conditions on contributions.

We also replicated the effect of common knowledge on contributions found in Studies 1 and 2 as well as the mediation models from Study 2 that found that the number of predicted group contributors, and certainty in those predictions, mediated the effect of common knowledge on cooperation. This provides stronger support that uncertainty about other agents' cooperative behavior underlies the prosocial effect of common knowledge on cooperation. Consistent with the results from Study 2, we again failed to replicate the interaction between information 
condition and threshold level observed in Study 1, suggesting that initial interaction effect might have been spurious.

\section{General Discussion}

The goal of this project was to investigate how common knowledge promotes cooperation, testing the hypothesis that common knowledge increases cooperation by reducing uncertainty about others' cooperative behavior. Introducing thresholds to the PGG transforms the game from a pure social dilemma to an anti-coordination or coordination problem, and because common knowledge increases coordination (Thomas et al., 2014; Thomas et al., 2018), we predicted that common knowledge would increase contributions by decreasing the uncertainty surrounding whether other group members will contribute. In three studies, we manipulated the information participants had regarding what their group members knew about the threshold, as well as the level of threshold needed to receive the public good. We found that common knowledge of the threshold increased cooperation in the PGG and that the effect of common knowledge was mediated by the predicted number of group members contributing and certainty about the predicted number of contributors.

Overall, our finding that common knowledge increased contributions supports recent work suggesting that common knowledge is an important mechanism for coordinating behavior (Thomas et al., 2014; Thomas et al., 2018; De Freitas et al., 2019). Our work builds upon this literature by showing that common knowledge not only increases cooperation in two-player coordination games, but that it can also increase cooperation in $n$-person coordination games that more closely model the kinds of cooperation problems we encounter in everyday life. More generally, this finding provides some support for the special role of common knowledge in human cooperation, and its function as a potentially distinct cognitive mechanism that may have evolved to help us solve coordination problems and for social strategizing (De Freitas et al., 2019; Thomas et al., 2016). Future work should continue to investigate the role common knowledge plays in coordination problems, as well as what role, if any, common knowledge plays in social dilemmas, where there is a conflict between an actor's self-interest and the interest of the group. If common knowledge evolved as an adaptation for social strategizing (Thomas et al., 2014), then it might actually reduce cooperation in social dilemmas where actors' interests are diametrically opposed to one another. Indeed, Thomas et al., (2016) found that common knowledge reduces prosocial helping in a bystander intervention task that models the volunteer's dilemma, a type of anti-coordination 
game. However, in our intermediate threshold PGGs, which model anti-coordination problems, we find a prosocial effect of common knowledge This tension suggests that more work is needed to better understand whether common knowledge will reduce prosocial behavior in a prisoner's dilemma or other social dilemma where cooperation is personally costly.

It is important to note that the focus of our knowledge conditions differed from the Thomas et al. (2014) study which examined common knowledge of the joint-payoff for coordinating. In our study, participants had common knowledge of the threshold, rather than the payoff. However, we believe our findings would extend to other important features of the PGG, such that common knowledge of the contribution multiplier, for example, would also increase contributions relative to private knowledge. Additionally, while we only compared common knowledge to private knowledge here, it would be interesting to examine shared or asymmetric knowledge (such as secondary or tertiary knowledge) of a threshold to see how it influences cooperation relative to common knowledge. Future work should compare common knowledge to shared knowledge of a threshold and explore whether common knowledge of other aspects of the game increases contributions.

One of our most important findings was that certainty about others' cooperative behavior mediated the effect of common knowledge about the threshold on contributions. In other words, when deciding whether to contribute, participants incorporated information about others' mental states in order to infer the likelihood that they would contribute and the threshold would be met. That is, common knowledge increased cooperation because it increased certainty that other group members would contribute and the threshold would be met. To the best of our knowledge, this finding provides the first evidence that common knowledge promotes coordination primarily by reducing uncertainty about the behavior of other social agents. This is consistent with past work showing that uncertainty about the threshold or game structure is generally detrimental to the provision of the public good and coordination (Dannenberg et al., 2011; McBride, 2010; Rubinstein, 1989). More generally, this finding highlights the important role our beliefs about other agents' beliefs and behavior play in our own cooperative behavior.

One question that arose after Study 1 is why contributions were not significantly lower when there was common ignorance of the threshold as compared to common knowledge. In Study 3 we sought to address this question: we found that participants were significantly more likely to contribute when there was common knowledge than common ignorance of the threshold. That the difference between conditions was significant in Study 3 but not in Study 1 is likely due to statistical noise between studies. However, in conjunction, the studies suggest there is a small, but real difference between common knowledge and common ignorance 
on contributions. Additionally, we found that-as with common knowledgecontributions in the common ignorance conditions were predicted by predicted contributors and certainty in those predictions. That contributions were similar when there was common ignorance or private knowledge of the threshold likely reflects the fact that participants in the common ignorance condition possessed common knowledge that there was $a$ threshold, just one of unknown size. So, although they lacked the specific threshold information, participants could still be reasonably certain that their group members knew there was a threshold and that they would contribute, which in turn motivated their own contribution decisions. Overall, our finding here that contributions were higher when there was common knowledge than common ignorance supports past work finding that uncertainty about the threshold size is generally detrimental to the provisioning of the public good (Barrett and Dannenberg, 2014; Dannenberg et al., 2015).

Our results also support past work that has found that thresholds promote cooperation in the PGG (Jordan et al., 2017; Szolnoki \& Perc, 2010; van de Kragt et al., 1983). Indeed, in both of our studies, participants contributed significantly more when there was a threshold, regardless of the threshold size or beliefs about others' knowledge of it. Our finding from Study 1, that participants contributed more to the public good even when the threshold was unknown to themselves and their group, further underscores the effectiveness of thresholds: merely the presence of a threshold was enough to increase contributions. Interestingly, while we did not find an effect of threshold size on contributions across all three studies, we did find that threshold size predicted the expected number of group members contributing: the higher the threshold, the more group members were predicted to contribute. It is surprising that predicted contributors and contribution decisions dissociated when looking at the effect of threshold size, as we otherwise found a strong predictive relationship between predicted contributors and contribution decisions. Future work should examine this finding in more depth. Overall, these findings demonstrate the important role thresholds have in bolstering cooperation, even when there is uncertainty about the size of, or information other individuals know about, the threshold.

There were a few limitations in our studies and opportunities for future work. One concern with our approach is that contribution decisions were binary, allor-nothing decisions rather than continuous contributions (e.g., any amount from $\$ 0$ to $\$ 0.30$ ). While our approach is not novel, previous work has used binary contribution decisions in threshold PGGs (Rapoport \& Eshed-Levy, 1989; van de Kragt et al., 1983), it is possible that people might behave differently when contributions are continuous. Data from our group supports this idea; in a previous study we found that threshold level influences contributions when they are continuous (blinded for peer review, in prep). We note that across both studies a 
number of participants were excluded for failing comprehension checks. We believe this high exclusion rate reflects the relatively complex nature of the threshold PGG and our inclusion of extensive comprehension checks-three on the dynamics of the threshold PGG and four on the information conditions (see the Supplement for the specific comprehension checks). We wish to note, however, that rates of exclusions were roughly similar across conditions (SOM - S11-13), suggesting that while the task itself was relatively complex, these exclusions are unlikely to have contributed to our reported effects.

Lastly, we would like to note that the effects of common knowledge on contributions found across our studies constituted a relatively small effect. We believe this could be due to several non-mutually exclusive reasons. First, our common knowledge manipulation was relatively subtle compared to how common knowledge is likely established in daily life as it was constrained by collecting data online; given a stronger, more naturalistic common knowledge manipulation (such as establishing common knowledge through eye contact) we would expect a significantly larger effect of common knowledge on cooperation. Second, we observed overall very high levels of cooperation (74\% across all studies) that may have constituted something of a ceiling effect, reducing movement between conditions. Third and finally, it is possible that the small stake size used here contributed to the overall high levels of cooperation and, by reducing the risk associated with contributing, lessened the effect of common knowledge. However, previous work has found that there is not a meaningful difference between large and small stake sizes (Amir et al., 2012). Future work should examine the boundary conditions of the effect of common knowledge on cooperation by manipulating common knowledge in a more naturalistic way, such as through eye contact and increasing the riskiness of contributions by varying the stake size and other factors.

In sum, we investigated whether common knowledge increases contributions in the PGG by reducing uncertainty about others' cooperative behavior. Across two studies, we found that common knowledge increased contributions in the PGG, and that this effect was mediated by the predicted number of group contributors and certainty about those predictions. These findings provide strong evidence that the effect of common knowledge on coordination is mediated by certainty about others cooperative behavior. Lastly, our studies provide the important insight that common knowledge can increase cooperation in $n$-person coordination problems. More generally, our results are consistent with theories that common knowledge is an evolved, cognitive mechanism for solving coordination problems. At the broadest level, our findings reveal the potential that common knowledge holds for promoting cooperation in the large-scale coordination problems that predominate our social lives. 
In print at Evolution \& Human Behavior

\section{Open Practices}

This project was pre-registered prior to data collection [Study 1:

http://aspredicted.org/blind.php?x=63ct9y; Study 2:

https://osf.io/brqky/?view only=ab7c7982f7454e439ca63c5806c00d52; Study 3:

https://osf.io/xqyjp/?view_only=7605805b183e470daafc2d7d0a535c88]. All of

our data and code are available in an online repository here

[https://osf.io/wkcrf/?view_only=6be9f35572bd44dc8927d75fd63292b0].

\section{Acknowledgments}

The authors thank the John Templeton Foundation for grant 61061 awarded to K.M. as well as the Cooperation Lab for helpful feedback on all stages of the research process. 


\section{References}

Almaatouq, A., Krafft, P., Dunham, Y., Rand, D. G., \& Pentland, A. (2020). Turkers of the world unite: Multilevel in-group bias among crowdworkers on amazon mechanical turk. Social Psychological and Personality Science, 11(2), 151159.

Amir, 0., \& Rand, D. G. (2012). Economic games on the internet: The effect of $\$ 1$ stakes. PloS one, 7(2), e31461.

Andrews, T. M., Delton, A. W., \& Kline, R. (2018). High-risk high-reward investments to mitigate climate change. Nature Climate Change, 8(10), 890.

Archetti, M., \& Scheuring, I. (2012). Game theory of public goods in one-shot social dilemmas without assortment. Journal of Theoretical Biology, 299, 9-20.

Arechar, A. A., \& Rand, D. (Preprint). Turking in the time of COVID.

Axelrod, R., \& Hamilton, W. D. (1981). The evolution of cooperation. Science, 211(4489), 1390-1396.

Balakrishnan, J. D., \& Ratcliff, R. (1996). Testing models of decision making using confidence ratings in classification. Journal of Experimental Psychology: Human Perception and Performance, 22(3), 615.

Baltag, A., Moss, L. S., \& Solecki, S. (2016). The logic of public announcements, common knowledge, and private suspicions. In Readings in Formal Epistemology (pp. 773-812). Springer, Cham.

Barrett, S., \& Dannenberg, A. (2014). Sensitivity of collective action to uncertainty about climate tipping points. Nature Climate Change, 4(1), 36-39.

Bradley, J. V. (1981). Overconfidence in ignorant experts. Bulletin of the Psychonomic Society, 17(2), 82-84.

Cadsby, C. B., \& Maynes, E. (1999). Voluntary provision of threshold public goods with continuous contributions: experimental evidence. Journal of Public Economics, 71(1), 53-73.

Clark, H. H., \& Marshall, C. R. (1981). Definite knowledge and mutual knowledge. Clutton-Brock, T. (2009). Cooperation between non-kin in animal societies. Nature, 462(7269), 51.

Dannenberg, A., Löschel, A., Paolacci, G., Reif, C., \& Tavoni, A. (2015). On the provision of public goods with probabilistic and ambiguous thresholds. Environmental and Resource Economics, 61(3), 365-383.

De Freitas, J., Thomas, K., DeScioli, P., \& Pinker, S. (2019). Common knowledge, coordination, and strategic mentalizing in human social life. Proceedings of the National Academy of Sciences, 116(28), 13751-13758.

Deutchman, P., Amir, D., Jordan, M., \& McAuliffe, K. (In prep). The Effect of Social and Non-Social Thresholds in the PGG. 
In print at Evolution \& Human Behavior

Diekmann, A. (1985). Volunteer's dilemma. Journal of Conflict Resolution, 29(4), 605-610.

Fehr, E., \& Fischbacher, U. (2004). Social norms and human cooperation. Trends in Cognitive Sciences, 8(4), 185-190.

Fehr, E., \& Gachter, S. (2000). Cooperation and punishment in public goods experiments. American Economic Review, 90(4), 980-994.

Fischbacher, U., Gächter, S., \& Fehr, E. (2001). Are people conditionally cooperative? Evidence from a public goods experiment. Economics letters, 71(3), 397-404.

Halpern, J. Y., \& Moses, Y. (1990). Knowledge and common knowledge in a distributed environment. Journal of the ACM (JACM), 37(3), 549-587.

Hauert, C., \& Doebeli, M. (2004). Spatial structure often inhibits the evolution of cooperation in the snowdrift game. Nature, 428(6983), 643.

Heizer, R. F. (1953). Aboriginal fish poisons. Bureau of American Ethnology Bulletin.

Horton, J. J., Rand, D. G., \& Zeckhauser, R. J. (2011). The online laboratory: Conducting experiments in a real labor market. Experimental economics, 14(3), 399-425.

Jansson, F., \& Eriksson, K. (2015). Cooperation and shared beliefs about trust in the assurance game. PloS One, 10(12), e0144191.

Jordan, J., McAuliffe, K., \& Rand, D. (2016). The effects of endowment size and strategy method on third party punishment. Experimental Economics, 19(4), 741-763.

Jordan, M. R., Jordan, J. J., \& Rand, D. G. (2017). No unique effect of intergroup competition on cooperation: non-competitive thresholds are as effective as competitions between groups for increasing human cooperative behavior. Evolution and Human Behavior, 38(1), 102-108.

Marks, M. B., \& Croson, R. T. (1999). The effect of incomplete information in a threshold public goods experiment. Public Choice, 99(1-2), 103-118.

McBride, M. (2010). Threshold uncertainty in discrete public good games: An experimental study. Economics of Governance, 11(1), 77-99.

Rand, D. G., \& Nowak, M. A. (2012). Evolutionary dynamics in finite populations can explain the full range of cooperative behaviors observed in the centipede game. Journal of Theoretical Biology, 300, 212-221.

Rapoport, A., \& Eshed-Levy, D. (1989). Provision of step-level public goods: Effects of greed and fear of being gypped. Organizational Behavior and Human Decision Processes, 44(3), 325-344.

Rubinstein, A. (1989). The electronic mail game: Strategic behavior under "almost common knowledge." The American Economic Review, 79, 385-391

Schelling, T. (1960). The Strategy of Conflict. Cambridge and London: Harvard University Press 
Sekhon, Jasjeet S. 2011. Multivariate and Propensity Score Matching Software with Automated Balance Optimization: The Matching package for R. Journal of Statistical Software. 42(7): 1-52.

Skyrms B. The Stag Hunt and the Evolution of Social Structure. Cambridge: Cambridge University Press; 2003.

Szolnoki, A., \& Perc, M. (2010). Impact of critical mass on the evolution of cooperation in spatial PGGs. Physical Review E, 81(5), 057101.

Thomas, K. A., DeScioli, P., Haque, O. S., \& Pinker, S. (2014). The psychology of coordination and common knowledge. Journal of Personality and Social Psychology, 107, 657-676

Thomas, K. A., De Freitas, J., DeScioli, P., \& Pinker, S. (2016). Recursive mentalizing and common knowledge in the bystander effect. Journal of Experimental Psychology: General, 145, 621- 629.

Thomas, K. A., DeScioli, P., \& Pinker, S. (2018). Common knowledge, coordination, and the logic of self-conscious emotions. Evolution and Human Behavior, 39, 179-190.

Thunström, L., Nordström, J., \& Shogren, J. F. (2015). Certainty and overconfidence in future preferences for food. Journal of Economic Psychology, 51, 101-113.

West, S. A., Griffin, A. S., \& Gardner, A. (2007). Social semantics: altruism, cooperation, mutualism, strong reciprocity and group selection. Journal of Evolutionary Biology, 20(2), 415-432.

Wit, A., \& Wilke, H. (1998). Public good provision under environmental and social uncertainty. European Journal of Social Psychology, 28(2), 249-256.

Van de Kragt, A. J., Orbell, J. M., \& Dawes, R. M. (1983). The minimal contributing set as a solution to public goods problems. American Political Science Review, $77(1), 112-122$. 